\title{
Does policy uncertainty threaten renewable energy? Evidence from G7 countries
}

\author{
Khalid Khan ${ }^{1}$ (1) $\cdot$ Chi Wei Su ${ }^{2}$ \\ Received: 24 August 2021 / Accepted: 21 September 2021 / Published online: 18 January 2022 \\ (C) The Author(s), under exclusive licence to Springer-Verlag GmbH Germany, part of Springer Nature 2021
}

\begin{abstract}
This study evaluates economic policy uncertainty (EPU) impact on renewable energy (RE) in the G7 countries. The finding explores a negative impact of EPU on RE across all quantiles, suggesting that EPU disturbs the macroeconomy, which leads to the decline in RE. However, the impact occurs in the upper quantiles, which recommends that high EPU influences RE rapidly. The coefficients show the varying effects of EPU on RE, as the impact of EPU decreases in Germany when the relationship changes from short to long term. Similarly, the impact increases in Italy, Japan, the UK, and the USA when the relationship changes from short to long run. The sustainable development of RE requires greater economic stability. This is possible if the government makes future policies by involving all stakeholders. Complete information about the planning, implementation, and modification of economic policies should be readily shared with all participants.
\end{abstract}

Keywords Economic policy uncertainty $\cdot$ Renewable energy $\cdot$ Environmental issues $\cdot$ Quantile on quantile $\cdot$ Wavelet transforms

JEL Codes Q40 $\cdot \mathrm{Q} 41 \cdot \mathrm{B} 4$

\section{Introduction}

Energy is the backbone of global economic development, has strategic significance, and is extremely important to a country (Cao et al. 2020). The existence of human societies throughout the world needs a constant supply of various energy sources. Access to various energy types has become more essential because of the rapid population growth and living standard (Zhu et al. 2020; Tao et al. 2021a). Moreover, environmental issues and energy security risks have increased the importance of renewable energy (RE) and is considered a key step towards achieving the ambitious goal of reducing carbon emissions (CE)(Hagspiel et al. 2021). In the past decade, RE

Responsible Editor: Nicholas Apergis

Khalid Khan

khalid.khan665@gmail.com

Chi Wei Su

cwsu7137@gmail.com

School of Finance, Qilu University of Technology, Jinan, China

2 School of Economics, Qingdao University, Qingdao, China has experienced significant development under the impetus of innovation, competition, and policy support. Similarly, largescale renewable technology advancements and rapid cost reductions are playing a leading role in the energy transition (Rechsteiner 2021). RE promotes energy security, environmental sustainability, and economic growth $\mathrm{Su}$ et al. 2021a). It is an important part of energy security, and a sharp decline in the production cost and policy support has driven the sustainable growth of RE (IEA 2017). The deregulation of the global electricity market involves the private sector, and the profit maximization goal determines RE investment (Abadie and Chamorro 2014; Tao et al. 2021b). Many countries have expanded supporting policies to maintain competition and investment in RE, which may be vulnerable to economic policy uncertainty (EPU). It is one of the key factors for the lack of development of RE because sudden policy changes have a considerable impact on RE. This study investigates whether EPU threatens RE growth in the group of 7 (G7) countries.

The G7 countries comprise Canada, France, Germany, Italy, Japan, the UK, and the USA and are the wealthiest nations; their total gross domestic product (GDP) accounts for $40 \%$ of the world and produces the largest emissions. Therefore, these countries are more responsible 
for reducing greenhouse gas emissions and leading the energy transition. Similarly, the G7 nations support netzero greenhouse gas emissions by 2050 and revise their 2030 climate goals, and countries have increased RE shares in their energy mix. These countries have provided substantial support for RE, which can be affected by frequent economic and political events which result in EPU. Moreover, in order to overcome environmental risks and maintain sustainable economic development, the energy transition process has received more attention (Wei et al. 2021). Tax reforms, international trade, and energy policies in these countries may affect RE and its development. Similarly, the feed-in tariff system in some of these countries has set the deadline for the public to sell RE, which promotes the production of renewable energy, and EPU may interfere with its implementation (Chen et al. 2019; Tao et al. 2021c). The nuclear accident in 2011 has triggered a more rapid transformation and the phasing out of nuclear energy. RE growth is threatened by EPU, and its expansion has been slow after 2014, unable to produce electricity that can cope with climate change (Dalby et al. 2018). These countries are the most affected by the coronavirus (COVID-19) pandemic and their economies have been contracted. The crisis has affected RE industries considerably because of lockdown, declining the demand for fuel and electricity. It has caused the delay of new projects and plants of renewable energy. Similarly, the sectors experience financial constraints, and investors have adopted the risk aversion policy, which has shrunk future investments. The investment declines in RE in France, Germany, the UK, and the USA as a result of the pandemic uncertainty. Similarly, a ban is imposed on nonessential activities which leads to the halting of the production of the major companies such as the Siemens Gamesa Renewable Energy and Vestas. The pandemic delays the underdevelopment renewable projects. Moreover, the lockdown leads to the closure of the various manufacturing plants for renewable energy technologies (RETs) which may slow down RE development.

The primary purpose of this research is to investigate the challenges EPU poses to RE in the G7 countries with a high level of income and economic development. These are the most advanced and influential countries with a high level of income and economic development, leading the world in economic development, consuming nearly $35 \%$ of global energy production, and contributing $32 \%$ of carbon emissions (Pirgaip and Dinçergök 2020). Likewise, it is a group of countries with coordinated economic activity that policy coordination becomes very essential (Monfort et al. 2003). The high economic status enables the G7 countries to follow energy conservation policies without disturbing their economies. The studies about EPU are limited in the aspect of RE, while the rising EPU concerns for macroeconomic indicators consumption, development, and investment in RE. The rising EPU prompts to delay or postpone the investment decision because of weak public and financial policies during the uncertainty (Adedoyin and Zakari 2020). Similarly, the rising EPU may have a negative impact on the economy, leading to the withdrawal of support and reduction of subsidies for RE development. Most of these countries have provided support to develop RETs that are highly susceptible to policy changes. The higher environmental issues have prompted these countries to reduce greenhouse gas emission levels by 2030 . However, EPU may challenge the role of the G7 countries in the overall decarbonization cooperation of the global economy through investment channels (Livingston 2016; Sun et al. 2021).

We can draw several contributions from this research. First, we study the response of RE to changes in the economic policies of these countries, as the G7 countries possess the most influential position in world economic affairs and play a leading role in the energy transition. After the Paris Agreement of 2015, these countries have a major responsibility to be the first to achieve their goals and make the world cleaner and safer. However, the road to achieving these ambitious goals is a daunting task, and some obstacles may be encountered, and EPU is one of the most important one (Burns 2019; Su et al. 2021b). Economic policies set the framework for a successful transition and encourage investors to switch to RE. These countries are considered being pioneers in the renewable energy market, and RETs have received substantial government support and subsidies. However, policy changes have adversely affected the RE market and investment decisions. The results support our notion that rising EPU has a negative impact on RE in the G7 countries. Second, as far as we know, this is the first study that uses the wavelet quantileon-quantile (QQ) method to evaluate the impact of EPU on $\mathrm{RE}$ in the $\mathrm{G} 7$ countries. It provides a macro correlation of the determinants across quantiles. Therefore, the results confirm that EPU has a negative impact on RE in the middle to high quantiles, which offers useful policy implications. Lastly, the novel method of wavelet quantile on quantile is another contribution of the current research to contemporary literature. This method combines wavelet transform and QQ program to study the nonlinear correlation of variables in different frequency domains. The results show that EPU has a negative impact on renewable energy in all quantiles, indicating that EPU will have an adverse impact on the macroeconomy, which will hinder investment and ultimately lead to a decline in renewable energy growth.

We structure the paper as follows. We review the previous literature in "Literature review," which follows the wavelet quantile-on-quantile approach in the "Methodology" section. Whereas we explain the data in the "Data" section, which is followed by an empirical 
analysis in "Empirical results." "Conclusion and policy implications" concludes this study.

\section{Literature review}

Fabrizio (2013) investigated the regulatory EPU effect on firms' investment in RE and confirmed that regulatory uncertainties reduce RE investment. Chronopoulos et al. (2016) found that investment in RE is subject to random provision and retraction of subsidy. The rising number of policy interventions decreases the size of RE projects. Dalby et al. (2018) showed the response of investment behavior caused by the risk of subsidy retraction. The results indicate that investors hold their investment when the policy changes increase and vice versa. Cao et al. (2020) found that uncertainties caused by the oil price have a significant impact on RE investment in China and led to the reduction of the firm's investment. Balcilar et al. (2019) investigated EPU effects on RE. They concluded that EPU adversely affects the macroeconomy that hampers investment and ultimately leads to affect RE growth. Moreover, the policymakers are hesitant to implement RE policies because of growing uncertainties. Burns (2019) concluded that investment in RE has been declining globally, and policy uncertainty is one of the fundamental factors. Sendstad and Chronopoulos (2020) showed that policy interventions have an adverse impact on RE investment timely. The retraction or provision increases or decreases the encouragement for investment. Appiah-Otoo (2021) analyzed EPU impact on RE during 2000-2018. The finding suggests that EPU has an insignificant negative influence on RE. Similarly, the results show that EPU and RE have no causality. Wei et al. (2021) found that EPU rapidly increases, which may affect the economic activities, and ultimately, this economic shock is reflected in RE.

Some studies about the G7 countries exist in the literature. Barradale (2010) evaluated the public EPU in the wind industry in the USA, employing survey data. The finding shows that rising EPU shrinks the investment in RE. Eryilmaz and Homans (2016) investigated EPU in the US investment decision in RE. The outcome suggests that EPU increases the threshold of the investment in RE. Harrabin (2016) showed that EPU deters essential investments in the UK energy system because of risks following unexpected interruptions and changes in subsidies. Gatzert and Vogl (2016) showed that policy risk is the main contributing factor in the risk return of RE investment in France and Germany. Ahmed et al. (2021) confirmed that both renewable energy research and development budgets, as well as EPU, are extremely volatile in the USA. Hagspiel et al. (2021) evaluated the green investment under uncertainty and concluded that investment in RE has been severely affected by the subsidy changes. Moreover, the investment is optimal if the likelihood of policy changes is low. Sohail et al. (2021) showed that monetary policy uncertainty has short- and long-term negative effects on RE in the USA. Liu et al. (2021) found that uncertainty caused by the COVID-19 has a significant impact on the returns and volatilities of RE stocks. Previous studies mostly cover the investment trends in RE. It concludes that the EPU effect passes through economic investment channels, which can disrupt RE. Furthermore, most of the aforementioned studies have examined the impact of EPU on investment in renewable energy and ignore RE production. The investment decision depends on the government policy framework, and frequent changes in the economic policy result in higher uncertainty, which discourages the investors. Thus, we analyze EPU impact on RE in different quantiles, which considers the renewable energy production in the G7 countries. This study employs the wavelet QQ methods which are suitable to investigate the nonlinear association across different quantiles. The finding suggests that EPU has a negative impact on RE in the medium to upper quantiles.

\section{Methodology}

\section{Wavelet analysis}

The use of wavelet analysis in economics is common ( $\mathrm{Su}$ et al. 2019b; Su et al. 2020). It is like a wave oscillation starting at zero and altering and reversing back to zero (Yahya et al. 2019). We use wavelets of different frequencies to fit the time series in the time and frequency domains (Graps 1995; Torrence and Webster 1999; Crowley 2007). We can construct dyadically the wavelet and converted a pair of particularly constructed functions $\theta$ and $\vartheta$ such that:

$\int \theta(t) d t=1$

$\int \vartheta(t) d t=0$

where $\theta$ and $\vartheta$ indicate the father wavelet and mother wavelet, respectively. The former identifies the smooth and lowfrequency components of the variable and later detects the comprehensive and high-frequency components of the variable. Hence, the achieved wavelet is demonstrated as follows based on the aforementioned equations.

$\theta_{u, v}(t)=2^{\frac{j}{2}} \theta\left(2^{u} t-v\right)$
$\vartheta_{u, v}(t)=2^{\frac{j}{2}} \vartheta\left(2^{u} t-v\right)$

The number of observations restricts the maximum number of scales that the scrutiny can measure $\left(T \geq 2^{u}\right)$.

A special feature of the wavelet expansion is the coefficient of the positioning attribute $\theta_{u, v}(t)$ which indicates the information content of the function at the estimated position $v 2^{-u}$ 
and frequency $2^{u}$. Thus, the $L^{2}(\mathbb{R})$ can be expanded underlying wavelet at the arbitrary level $u_{0} \in \mathbb{N}$ over different scales.

$X(t)=\sum_{v} R_{u_{0, v}} \theta_{u_{0, v}}(t)+\sum_{u>u o} \sum_{v} d_{u, v} \theta_{u, v}(X), u=u_{0}, \ldots, u$

where $\theta_{u o, v}$ represents a scaling function and the scale coefficients, where the corresponding coarse-scale coefficients $C_{u o}$, ${ }_{v}$ and $d_{u, v}$ mean the comprehensive coefficients stated by $C_{u o}$, ${ }_{v}=\int X(t) \theta_{j, k}(t) d t$ and $d_{u, v}=\int X(t) \vartheta(t) d t$, respectively. The series $C_{u, t}=\sum_{u} C_{u 0, u} \theta_{u o, v}(t)$ suggests a smooth form of the original series $X(t)$, which detects the long-run (i.e., low frequency) features, while the series $D_{u, t}=\sum_{k} d_{u, v} \vartheta_{u, v}(\mathrm{t})$ notices local variations (i.e., the higher frequency features) of $X(t)$.

\section{Maximum overlap discrete wavelet transform}

We perform the wavelet transform of the discrete sampled by discrete wavelet transform (DWT). It is based on the scaling filter $r\left(r l, l=0, \ldots, L-.1^{2}\right)$ and the wavelet filter $\left(s_{l}, l=0, \ldots\right.$, $L-1^{3}$ ). $L \in \mathbb{N}$ denotes the length of the filter (Percival and Walden 1997). The wavelet filter fulfills these three characteristics.

$\sum_{l-0}^{L-1} r_{l}=0, \sum_{l-0}^{L-1} r_{l}^{2}=0 \quad \sum_{l-0}^{L-1} r_{l} r_{l+2 n}=0 \forall \mathrm{n} \in \mathbb{N}$

The low- and high-pass filters are defined as quadrature mirror filters, which

$r_{l}=(-1)^{l} s_{L-1-l}$ or $s_{l}=(-1)^{l+1} r_{L-1-l}, l=0, \ldots, L-1$

Similarly, the scaling filter fulfills the conditions.

$\sum_{l-0}^{L-1} s_{l}=0, \sum_{l-0}^{L-1} s_{l}^{2}=0 \quad \sum_{l-0}^{L-1} s_{l} s_{l+2 n}=0 \forall \mathrm{n} \in \mathbb{N}$

The wavelet and scaling coefficients of DWT at the $p$ th level for $p \in\{1, \ldots, p\}$ are defined as:

$h_{j, t}=\sum_{l-0}^{L-1} r_{l} X_{t-1} \quad$ and $=\quad g_{j, t}=\sum_{l-0}^{L-1} s_{l} X_{t-1}$

The maximal overlap discrete wavelet transform suggested by Percival and Walden (1997) decomposes the series. This method is useful in solving the DWT limitations. Daubechies least asymmetric, as well as the scaling coefficients, achieves the wavelet as it has high power to detect time-scale deviation in a series.

We decompose the main data series into a different frequency band and a set of wavelet coefficients. The rescaled scaling is achieved by incorporating the MODWT as follows;

$\widetilde{j}_{p, l}=\frac{r_{p, l}}{2^{\frac{p}{2}}} \quad$ and $\quad k_{p, l}=\frac{s_{p, l}}{2^{p / 2}}, p=0, \ldots \ldots, p$
As recommended by Mallat (1989), $\widetilde{h}_{p, t}$ and $\widetilde{g}_{p, t}$ are achieved by using the pyramid algorithm. It requires three inputs for each iteration of the MODWT algorithm. The first starts by faltering data and gives the listed wavelet and scaling coefficients:

$\widetilde{h}_{1, t}=\sum_{l-0}^{L-1} \widetilde{r}_{l} X_{t-1}$ and $\widetilde{s}_{1, t}=\sum_{l-0}^{L-1} \widetilde{s}_{l} X_{t-1}$

The scaling coefficients of the first step develop as input data vectors and are used to achieve the second step. We illustrate the second-level wavelet as follows:

$\widetilde{h}_{2, t}=\sum_{l-0}^{L-1} \widetilde{r}_{l} \widetilde{g}_{1, t-l} \bmod \mathrm{N}$ and $\widetilde{g}_{2, t}=\sum_{l-0}^{L-1} \widetilde{s}_{l} X_{t-l} \bmod \mathrm{N}$

Likewise, the $p$ th-level MODWT wavelet and scaling coefficients of the time series $X(t)$ are expressed as:

$\widetilde{h}_{p, t}=\sum_{l-0}^{L-1} \widetilde{r}_{l} \widetilde{g}_{1, t-l} \bmod \mathrm{N}$ and $\widetilde{g}_{J, t}=\sum_{l-0}^{L-1} \widetilde{s}_{l} X_{t-l} \bmod \mathrm{N}$

\section{The quantile-on-quantile method}

We briefly explain the characteristic of the QQ method proposed by Sim and Zhou (2015) and specify the model to investigate EPU impact on RE. Several studies use for econometric analysis the novel approach for energy consumption, tourism analysis, and Islamic bond volatility. It is assumed to be the general form of the standard quantile regression model, employed for the investigation of the quantiles of a variable affecting the conditional quantiles of another variable. The approach is the aggregate of quantile regression and nonparametric estimation of local linear regression. Therefore, we use the QQ approach to examine the impact of the quantiles of EPU on the quantiles of RE. The procedure begins with the nonparametric quantile regression model.

$\mathrm{RE}=\alpha^{\vartheta}\left(\mathrm{EPU}_{t}\right)+\varepsilon_{t}^{\vartheta}$

We examine the association between the $\vartheta$ th quantile in the background of $\mathrm{EPU}^{\tau}$ by using local linear regression. As $\alpha^{\vartheta}($.) is unidentified, this function can be estimated by a first-order Taylor expansion about the quantile EPU ${ }^{\tau}$.

$\alpha^{\vartheta}\left(\mathrm{EPU}_{t-1}\right)=\alpha^{\vartheta}\left(\mathrm{EPU}^{\tau}\right)+\alpha^{\vartheta}\left(\mathrm{EPU}^{\tau}\right)\left(\mathrm{EPU}_{t-1}-\mathrm{EPU}^{\tau}\right)$

where $\alpha^{\vartheta}$ shows the partial derivative of $\alpha^{\vartheta}\left(E P U_{t-1}\right)$ in the context of EPU. The outcome is termed the marginal effect which can be construed as the slope coefficient in the linear regression model.

The parameters $\alpha^{\vartheta}\left(\mathrm{EPU}_{t-1}\right)$ and $\alpha^{\vartheta}\left(\mathrm{EPU}^{\tau}\right)$ are the conspicuous aspect of Eq. (13) which are doubly indexed in $\vartheta$ and $\tau$. Assume that $\alpha^{\vartheta}\left(\mathrm{EPU}_{t-1}\right)$ and $\alpha^{\vartheta}\left(\mathrm{EPU}^{\tau}\right)$ are both functions 
of $\vartheta$ and $\mathrm{EPU}^{\tau}$, and that $\mathrm{EPU}^{\tau}$ is a function of $\tau$, it is clear that both $\alpha^{\vartheta}\left(\mathrm{EPU}_{t}-{ }_{1}\right)$ and $\alpha^{\vartheta}\left(\mathrm{EPU}^{\tau}\right)$ are functions of $\theta$ and $\tau$. Furthermore, $\alpha^{\vartheta}\left(\mathrm{EPU}_{t}-{ }_{1}\right)$ and $\alpha^{\vartheta}\left(\mathrm{EPU}^{\tau}\right)$ can be retitled as $\alpha_{0}(\vartheta, \tau)$ and $\alpha_{1}(\vartheta, \tau)$, respectively. Therefore, Eq. (16) can be rephrased as:

$\alpha^{\vartheta}\left(E P U_{t-1}\right)=\alpha^{\vartheta}(\vartheta, \tau)+\alpha_{1}(\vartheta, \tau)\left(\mathrm{EPU}_{t-1}-\mathrm{EPU}^{\tau}\right)$

By replacing Eq. (14) into (16), we get (17)

$$
\underbrace{R E_{t}=\alpha_{0}(\theta, \tau)+\alpha_{1}(\vartheta, \tau)\left(\mathrm{EPU}_{t-1}-\mathrm{EPU}^{\tau}\right)}_{*}+\varepsilon_{t}^{\vartheta}
$$

where RE represents renewable energy. The part (*) of Eq. (17) is the $\vartheta$ th conditional quantile of EPU. However, unlike the function of the standard conditional quantile, this manifestation replicates the association between the $\vartheta$ th quantile of EPU and the $\vartheta$ th quantile of RE because the parameters $\alpha_{0}$ and $\alpha_{1}$ are doubly indexed in $\vartheta$ and $\tau$. Likewise, a linear relation is not supposed at any time between the quantiles of the studied variables.

Estimating Eq. (17) requires replacing $\mathrm{EPU}_{t-1}$ and $\mathrm{EPU}^{\tau}$ with their estimated counterparts, $\mathrm{EPU}_{t-1}$ and $\mathrm{EPU}^{\tau}$, respectively. The local linear regression estimates of the parameters $\sigma_{0}$ and $\sigma_{1}$, which are estimates of $\alpha_{0}$ and $\alpha_{1}$, respectively, are obtained by solving the following minimization problem:

$\min _{\sigma_{0}, \sigma_{1}} \sum_{i=1}^{n} \rho_{\theta}\left[\mathrm{RE}_{t}-\sigma_{0}-\sigma_{1}\left(\widetilde{\mathrm{EU}_{t-1}}-\widetilde{\mathrm{EPU}^{\tau}}\right)\right] \times K\left(\frac{F_{n}(\widetilde{\mathrm{EPU} \tau})-\tau}{h}\right)$

where $\rho \vartheta(\varepsilon)$ is the quantile loss function, defined as $\rho \vartheta(\varepsilon)=$ $\varepsilon(\vartheta-\mathrm{I}(\varepsilon<0))$, and $I$ indicates the usual indicator function. $K$ $(\cdot)$ represents the Gaussian kernel function, and $h$ represents the bandwidth parameter of the kernel.

\section{Data}

We analyze EPU impact on RE from 2000:01 to 2020:12 in the G7 countries. The energy transition occurs in the early 2000s, and spending on RETs is observed. These countries lead in the energy transition process coupled with the abundance of renewable resources, the rising cost of fossil fuels, and environmental risks. Similarly, some of these countries have invested heavily in RE, which are dominating players in the energy market. At the beginning of the new millennium, oil prices rise because of the depletion of oil fields, and the entry of new consumers from Asia increases the energy demand (Khan et al. 2021; Su et al. 2019a). However, this rising demand has both financial and environmental costs, and there is a discussion to convert towards RE to protect the environment and maintain energy supply for sustainable development (Wang et al. 2021; Khan et al. 2020a). On the other hand, EPU in these countries remains relatively low in the early 2000 s, which provides a conducive environment for RE growth. Moreover, the period has witnessed an unprecedented global economic boom which results in the rising energy demand and reflects in the enormous investment in RE. Thus, energy security, environmental issues, and low generation cost support the energy transition, which is vulnerable to EPU (Vakulchuk et al. 2020; Su et al. 2021c).

We decompose the series into short-, medium-, and longterm trends. The short-run reveals changes in RE caused by EPU in the short-term horizon (between 1 and 3 years). The medium-term horizon measures the variation between 4 and 5 years. Similarly, EPU impact on RE in the long-term horizon from 6 years onwards. Caldara and Iacoviell (2018) constructed the EPU ${ }^{1}$, which is a weighted average of national news articles deliberate EPU each month for these countries (Khan et al. 2021). RE is the sum of wind, solar, and hydropower, measured in terawatt hours, and are obtained from World Development Indicators (WDI) of the World Bank. Table 1 shows the summary statistics of EPU and RE. It shows that the UK has the highest EPU value, followed by France. Similarly, the standard deviation is highest for the UK, followed by Canada. The skewness values of EPU are positive for all the countries. The kurtosis values of EPU are greater than 3 for all countries, which suggests leptokurtic distribution. The Jarque-Bera test of EPU shows all countries are nonnormally distributed. The mean value of RE is highest for the USA followed by Canada. It evidences that these two countries are more developed and have higher energy demand. The environmental obligation results in the rapid energy transition in these countries. The standard deviation of RE for the USA is highest, which is followed by Canada. RE for all countries skewed to the right side except Canada. Similarly, the kurtosis values of RE platykurtic are distributed because it is less than 3. Last, the Jarque-Bera test evidence that RE is non-normally distributed for the G7 countries.

We illustrate the correlation coefficients between EPU and $\mathrm{RE}$ in the G7 countries in Table 2. The result shows that EPU and RE are highly positively correlated for all the countries. Moreover, the highest correlation coefficient is recorded for the UK, followed by Canada, France, and Italy. The correlations are highly significant, evident from the $p$-values which are statistically significant at the $1 \%$ level.

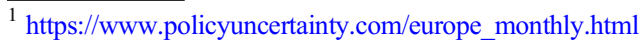


Table 1 Summary statistics

\begin{tabular}{|c|c|c|c|c|c|c|}
\hline Country & Variables & Mean & Std. dev. & Skewness & Kurtosis & $\mathrm{J}-\mathrm{B}$ \\
\hline \multirow[t]{2}{*}{ Canada } & EPU & 174.920 & 116.516 & 1.265 & 4.632 & $95.267^{* * * *}$ \\
\hline & $\mathrm{RE}$ & 389.728 & 31.658 & -0.040 & 1.604 & $20.509^{* * * *}$ \\
\hline \multirow[t]{2}{*}{ France } & EPU & 185.814 & 103.070 & 0.652 & 3.341 & $19.094^{* * *}$ \\
\hline & $\mathrm{RE}$ & 81.981 & 10.124 & 0.620 & 2.308 & $21.199^{* * * *}$ \\
\hline \multirow[t]{2}{*}{ Germany } & EPU & 146.220 & 78.403 & 1.512 & 6.091 & $196.446^{\text {**** }}$ \\
\hline & $\mathrm{RE}$ & 126.018 & 69.801 & 0.396 & 1.839 & $20.739^{* * * *}$ \\
\hline \multirow[t]{2}{*}{ Italy } & EPU & 112.592 & 40.781 & 0.786 & 3.793 & $32.589^{* * *}$ \\
\hline & $\mathrm{RE}$ & 80.210 & 28.480 & 0.151 & 1.321 & $30.549^{* * * *}$ \\
\hline \multirow[t]{2}{*}{ Japan } & EPU & 107.603 & 33.498 & 1.031 & 4.631 & $272.493^{* * * *}$ \\
\hline & $\mathrm{RE}$ & 156.942 & 38.220 & 1.028 & 2.797 & $44.880^{* * * *}$ \\
\hline \multirow[t]{2}{*}{ UK } & EPU & 212.339 & 158.210 & 1.658 & 7.917 & $369.337^{\text {**** }}$ \\
\hline & $\mathrm{RE}$ & 47.309 & 38.816 & 0.810 & 2.219 & $33.951^{* * * *}$ \\
\hline \multirow[t]{2}{*}{ USA } & EPU & 135.281 & 67.034 & 2.054 & 9.297 & $593.651^{\text {**** }}$ \\
\hline & $\mathrm{RE}$ & 485.354 & 158.369 & 0.713 & 2.360 & $26.789^{* * * *}$ \\
\hline
\end{tabular}

Note: ${ }^{* * *}$ denotes significance level at $1 \%$

\section{Empirical results}

We exhibit the QQ results of the original and decomposed series in Fig. 1 (A1-D1). The outcome of the original data shown in Fig. 1 (A1) is that the EPU of Canada has a negative impact on RE, and the impact is observed in the lower to middle quantile (0.45-0.60), which shows that rising EPU will decline RE development. The country has substantial RE sources because of its large area of diversified geography. However, the imprecise policies and lack of regulation for RE may cause uncertainty. The country has no national policy for greenhouse regulation which may be an obstacle to renewable development. Moreover, the greatest impact of EPU on RE appears in the lower to middle quantile, so low levels of EPU will cause a greater decrease in RE. Figure 1 (B1-D1) illustrates the wavelet decomposed series results. It shows that EPU has affected RE negatively in the upper quantiles

Table 2 Correlation between EPU and RE

\begin{tabular}{llll}
\hline & Correlation & $t$-value & $\begin{array}{l}p \text { - } \\
\text { value }\end{array}$ \\
\hline Canada & 0.734 & $17.108^{\text {*** }}$ & 0.000 \\
France & 0.600 & $11.858^{\text {*** }}$ & 0.000 \\
Italy & 0.585 & $11.47^{\text {*** }}$ & 0.000 \\
Germany & 0.400 & $6.901^{\text {**** }}$ & 0.000 \\
Japan & 0.177 & $2.856^{\text {**** }}$ & 0.004 \\
UK & 0.810 & $21.878^{* * *}$ & 0.000 \\
US & 0.487 & $8.830^{* * *}$ & 0.000 \\
\hline
\end{tabular}

Note: ${ }^{* * *}$ denotes the significance level at $1 \%$
$(0.70-0.80)$. The decomposed data result shows that EPU is harmful to RE development in all categories.

We examined the validity of the results through average QQ estimates and quantile regression, which is illustrated in Fig. 1 (a1-d1). It recommends that the impact of EPU on RE is consistently negative across all quantiles. The coefficients express that the size of the impact of EPU on RE decrease in the medium and long run. Similarly, the relationship becomes more regular in the wavelet decomposed series.

Figure 2 (A2) exhibits the results for France. The finding recommends that EPU has a negative impact on RE in the upper quantiles (0.70-0.80). The highest impact of EPU on RE illustrates that economic uncertainty is extremely unfavorable for renewable generation. The country produces $23 \%$ of its total energy from RE. However, the size of renewable share in the energy mix is relatively small, which can be easily affected or slowed down by the policy changes. The country has experienced several episodes of political and economic uncertainties which can easily impede RE. The decomposed data results are illustrated in Fig. 2 (B2-D2). It shows that EPU has a negative impact on RE in the upper quantiles (0.90-0.85). EPU has a disruptive effect on RE in the short run and may cause to threaten or slow down RE development. The regulatory uncertainty and unfavorable economic policy have slowed RE. However, in the medium run, EPU declines $\mathrm{RE}$ in the upper to medium quantiles $(0.70-0.50)$. The coefficient shows that EPU has a strong effect on RE, suggesting

Fig. 1 (A1-D1) QQ estimates of the slope coefficient. Note: The graph illustrates quantiles of EPU in the $x$ axis and the quantiles of RE in the $y$ axis. (a1-d1) Quantile regression and QQ estimates. Note: Quantile regression is represented by the black line, and QQ estimates are denoted by the red line 
(A1)

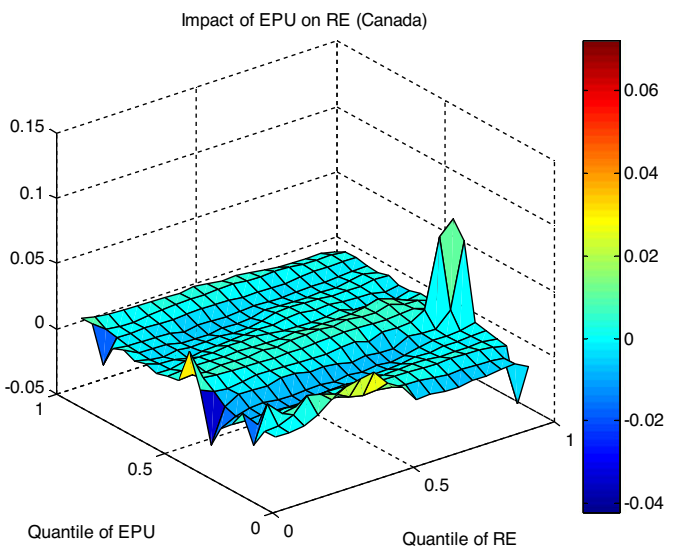

(C1)

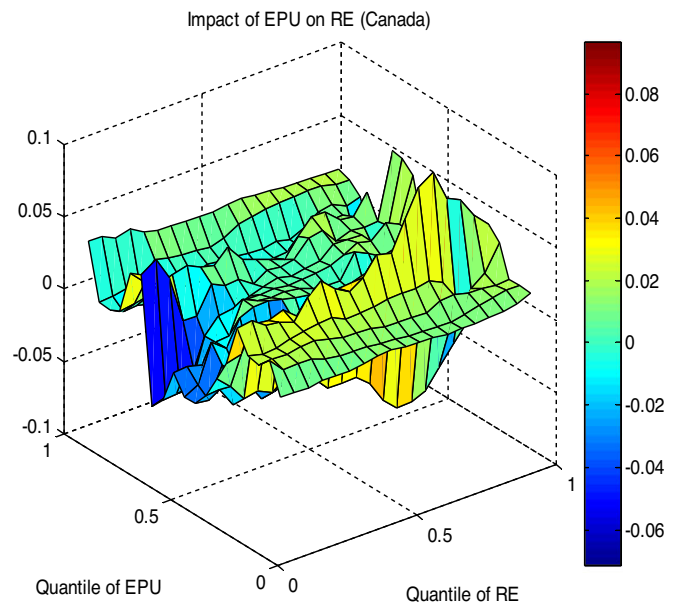

(a1)

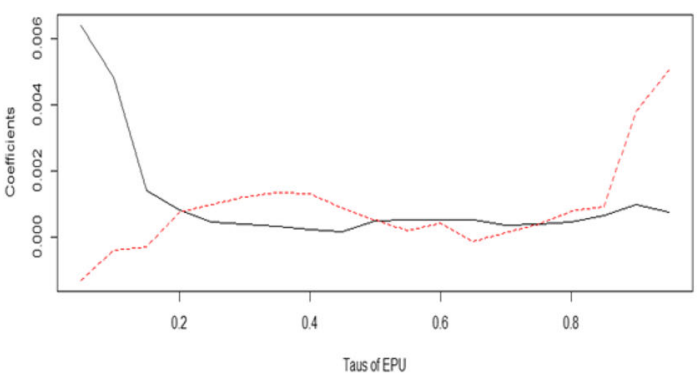

(c1)

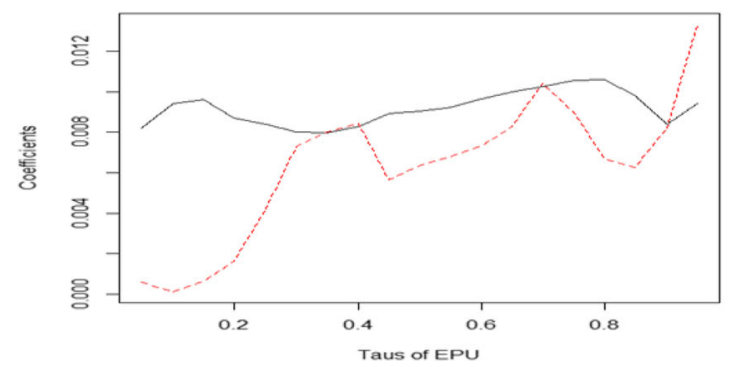

(B1)

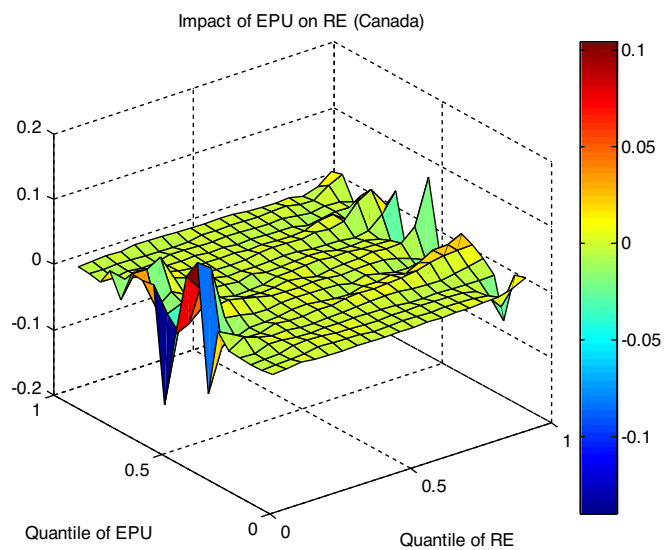

(D1)

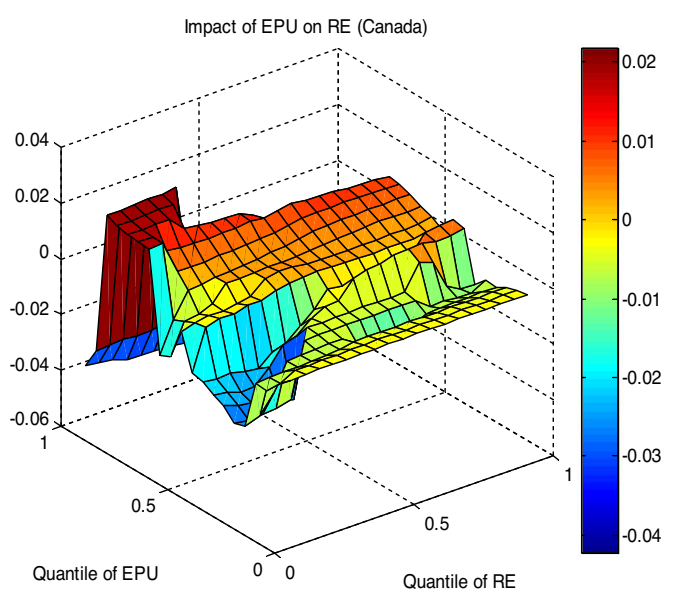

(b1)

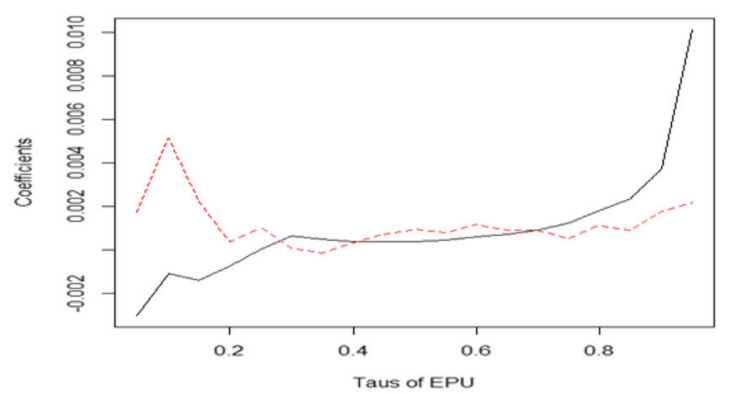

(d1)

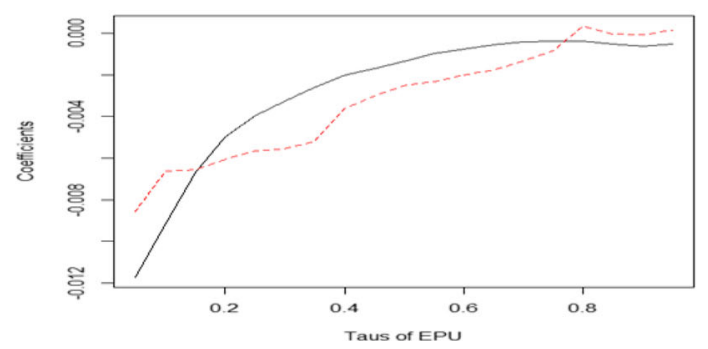


(A2)

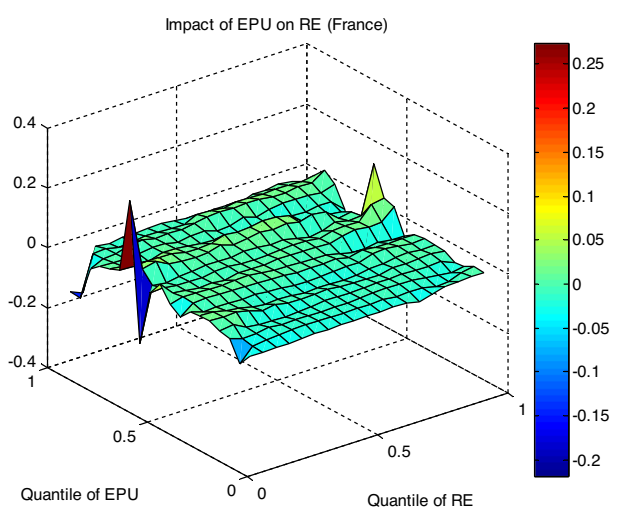

(C2)

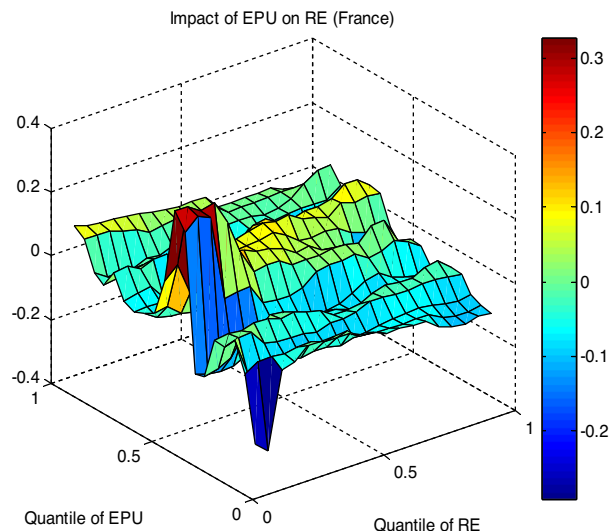

(a2)

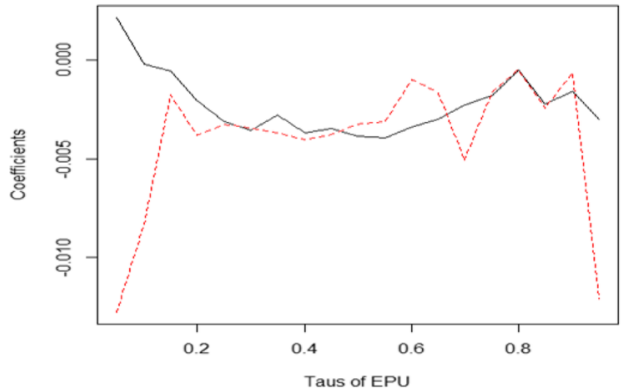

(c2)

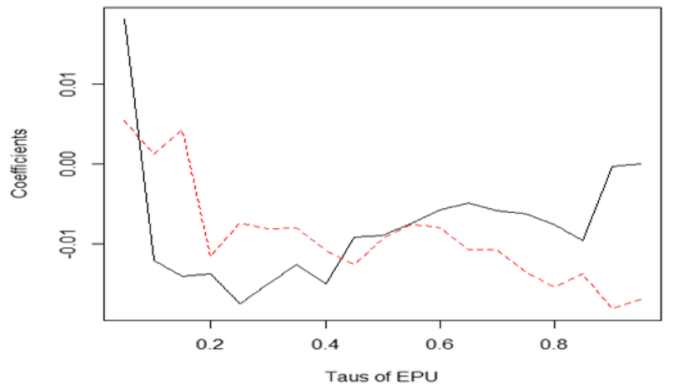

(B2)

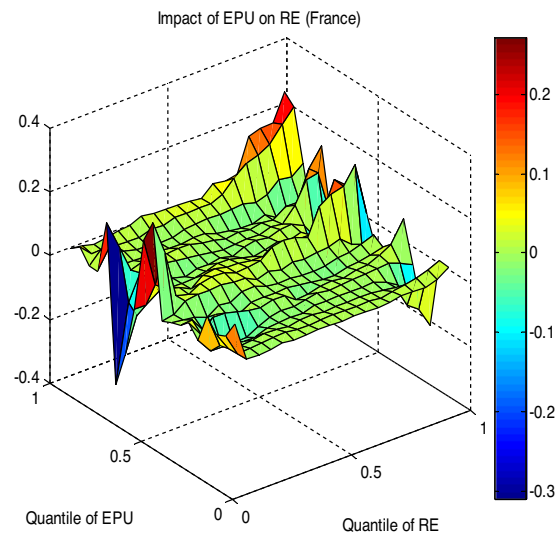

(D2)

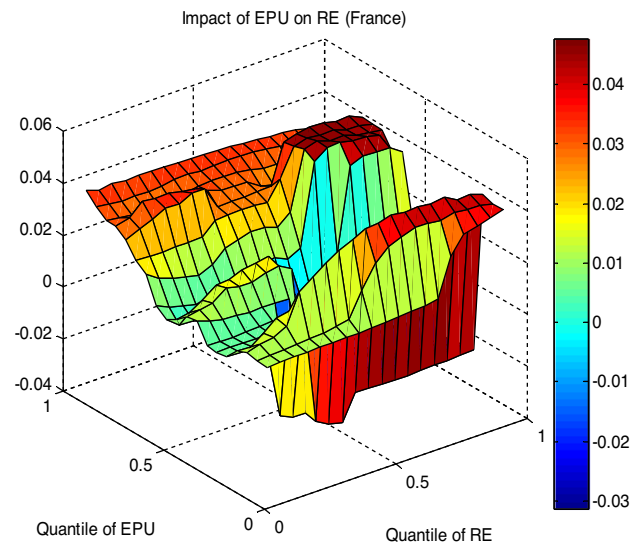

(b2)

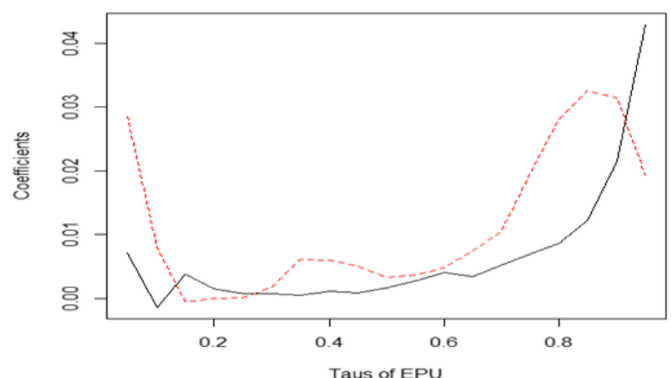

$(\mathrm{d} 2)$

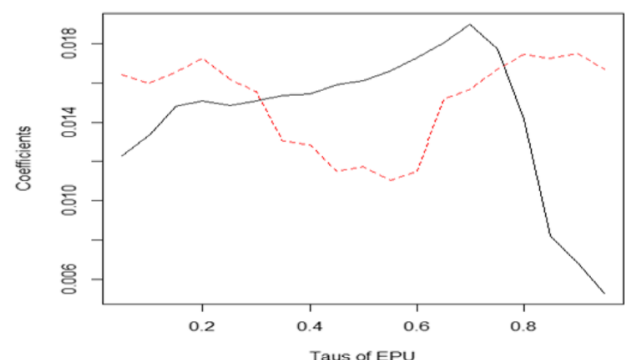

Fig. 2 (A2-D2) QQ estimates of the slope coefficient. (a2-d2) Quantile regression and QQ estimates 
that uncertainty more rapidly affects RE in the midterm. On the other hand, EPU has a positive impact on RE in the long run in the upper quantiles $(0.70-0.80)$.

We witness the validity of the results through QQ estimates and quantile regression, which is illustrated in Fig. 2 (a2-d2). It recommends that the impact of EPU on RE is negative in all quantiles. However, there is a positive impact of EPU on RE in the upper quantiles, suggesting that higher uncertainty may cause improve RE development and investment.

Figure 3 (A3) highlights the results for Germany. It shows that EPU has a negative impact on RE in the upper quantiles $(0.95-0.70)$ and the highest impact is detected, which suggests EPU will cause a greater decline. The country has a distinctive position in the G7 countries in the RE sector and produces a major portion of energy coming from RE. The country is the pioneer in the energy transition and has set up an ambitious plan to reduce greenhouse gas emissions by $40 \%$ in 2020 as compared to 1990. It is one of the successful cases of energy transition and produces $46 \%$ of the total energy from RE. The energy transition policy, known as Energiewende, provides an important framework for energy sector reforms (Rechsteiner 2021). The country has provided substantial support, which results in the significant investment in RE during 2005-2012. However, in the post-economic crisis, these subsidy schemes are curtailed to relieve the consumer electricity burden, which can be reflected in the decline of RE. The Renewable Energy Sources Act encourages RE by specifying feed-in tariffs and the market premium which the grid operator will have to pay for RE served by the power grid (Chen et al. 2019; Su et al. 2021d). It has fixed the period for the public to sell RE for 20 or 15 years, which pushes RE production. However, RE experiences challenge to halt the energy transition process through grid operators and discriminatory fees charged for grid connection (Rechsteiner 2021). The wavelet decomposed results are highlighted in Fig. 3 (B3-D3). The result shows that EPU declines RE in the short run in the upper quantiles (0.95-0.90), revealing that higher EPU translates into a higher decline in RE. Similarly, EPU has a negative impact on RE in the upper to lower quantiles $(0.80-0.40)$ in the mid-term, which suggests that higher uncertainty may have less impact on RE. Also, EPU has declined RE in the lower to upper quantiles $(0.40-0.80)$ in the long run, indicating that low EPU may rapidly decline RE. The coefficient shows that the impact on RE is greater in the short term, so policy shocks harm RE.

The validity results are shown in Fig. 3 (a3-d3). It confirms that the impact of EPU on RE is consistently negative in all quantiles, so higher uncertainty will adversely affect RE. However, compared with the short term, the intensity of the medium- and long-term impact has declined.

Figure 4 (A4) shows the Italy results. It shows that EPU has a negative influence on $\mathrm{RE}$ in the lower to medium quantiles (0.45-0.65). However, the highest impact of EPU on RE is (-
0.069), suggesting that EPU has an extensive impact on RE. There is a considerable increase in RE in the past decade driven by the European Union (EU) pressure and other environmental obligations. The rising trend in $\mathrm{RE}$ is observed during 2005-2011 because of lower costs and higher incentives provided by the government. In 2009, the country has adopted the climate change measures package and set the target for RE. The electricity from renewable sources reached a record in 2014 of around $43 \%$ of total gross electricity generation. The transition process encounters several political issues that hinder RE. Figure 4 (B4-D4) shows the wavelet decomposition result. It indicates that EPU has a negative impact on RE of the upper quantile $(0.85-0.80)$ in the short term, indicating that the larger the EPU, the greater the decrease in RE. However, EPU affects RE in the lower to medium quantiles $(0.15-0.60)$ in the mid-term, which explains the low level of uncertainty followed by a greater change in RE. Finally, in the long run, EPU has a negative impact on $\mathrm{RE}$ in the upper quantiles (0.60-0.70). The results explain that the size of impact increases when the relationship changes from short to medium and long, as seen from the coefficients.

The validity of the results is shown in Fig. 4 (a4-d4). It states that the impact of EPU on RE is consistently negative across all quantiles, and the coefficient increases from the lowmiddle quantile to the high-middle quantile. It shows that in the long run, EPU has a greater impact on RE than in the short run.

Figure 5 (A5) highlights the results for Japan. It shows that EPU has a negative effect on RE in the lower to upper quantiles (0.40-0.70). Energy policy is at a turning point triggered by the Fukushima nuclear power plant in 2011. Prior to this, the government's goal is to increase nuclear power by 2030, but the incident has led to changes in the structure of the energy mix. In the post-Fukushima period, nuclear power production decreased by $64 \%$ and thermal power plant production increased, which heightens the carbon emission (CE)(Zhu et al. 2020; Qin et al. 2021). The government has started a policy of replacing nuclear energy with RE and introduced a feed-in tariff system, forcing large companies to purchase energy produced by solar, wind, and hydropower at a fixed price. It helps to promote solar power generation; however, the rate is slow compared to other countries. The main reason is the cancellation of subsidies, and RE power generation facilities have become the target of the 2012 Environmental Impact Assessment Law. In addition, major companies refuse to allow operators to access the grid. The results of wavelet decomposition are shown in Fig. 5 (B5D5). According to the results, in the short term, EPU has a negative impact on RE in lower to upper quantiles $(0.45$ 0.80 ), showing that RE is more sensitive to moderate EPU. From the mid-term perspective, EPU has a negative impact on $\mathrm{RE}$ in the medium quantile (0.60-0.65), highlighting the 
(A3)

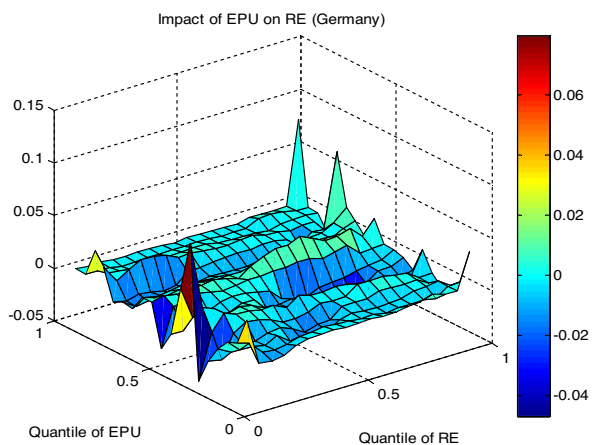

(C3)

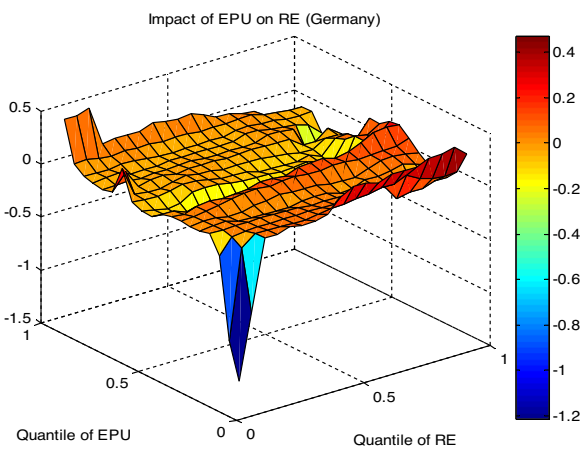

(a3)

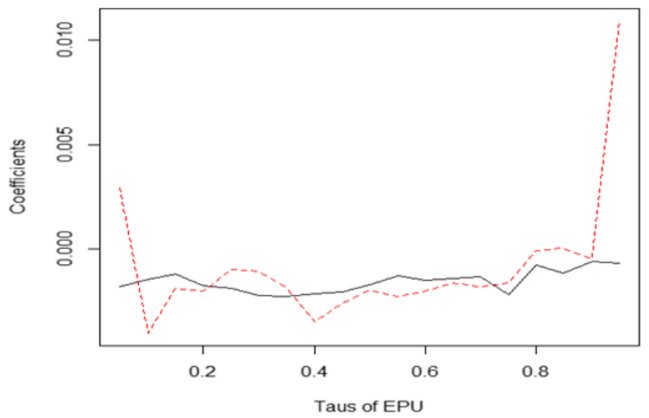

(c3)

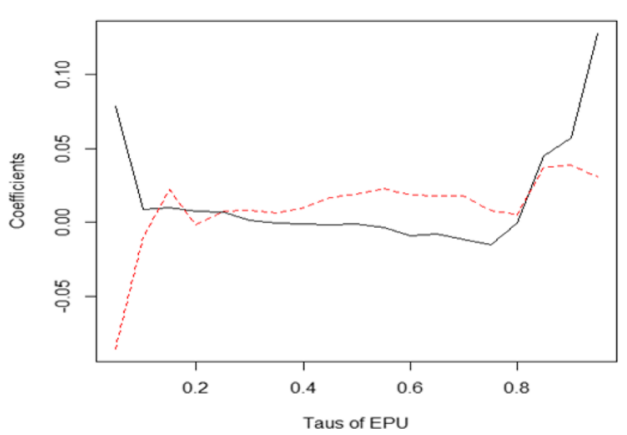

(B3)

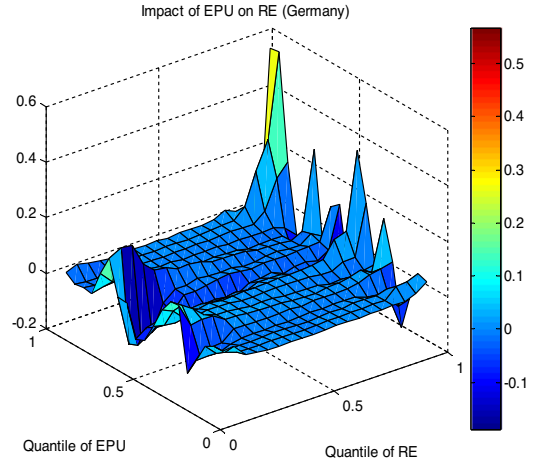

(D3)

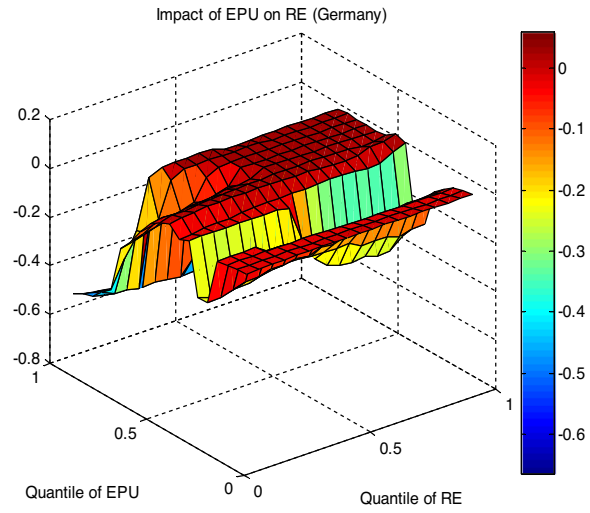

(b3)

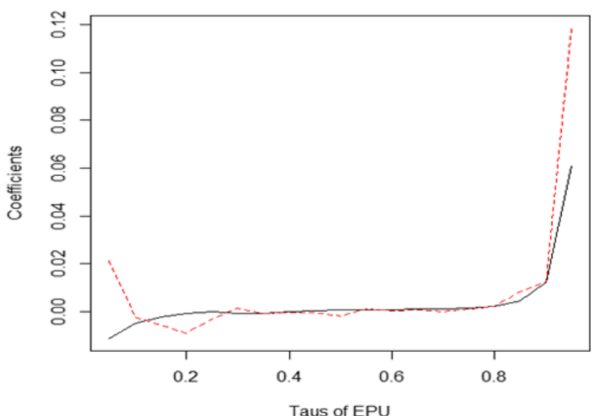

(d3)

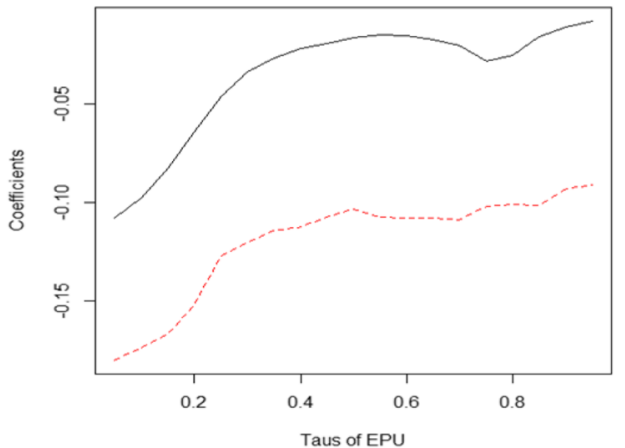

Fig. 3 (A3-D3) QQ estimates of the slope coefficient. (a3-d3) Quantile regression and QQ estimates 
(A4)

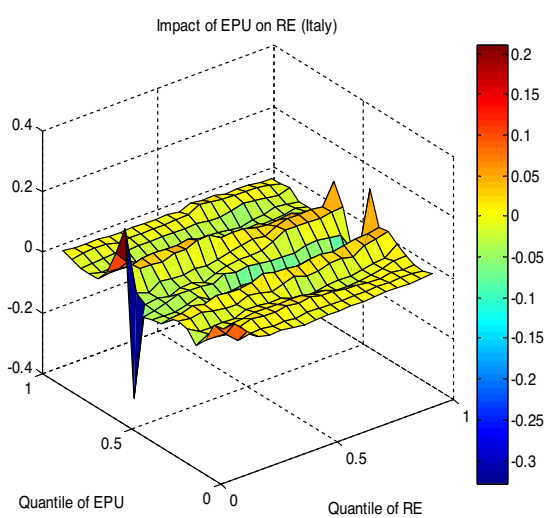

(C4)

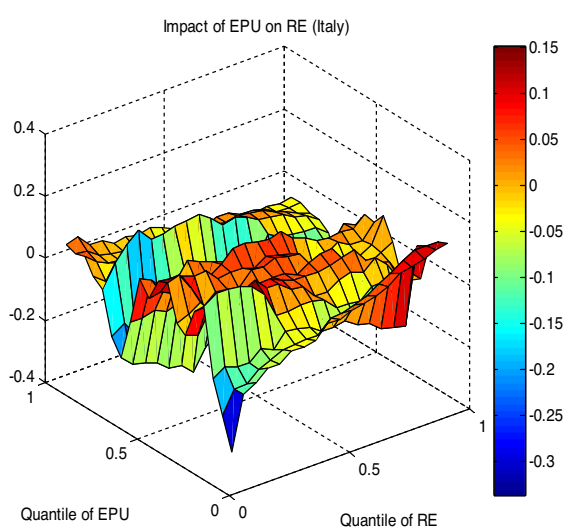

(a4)

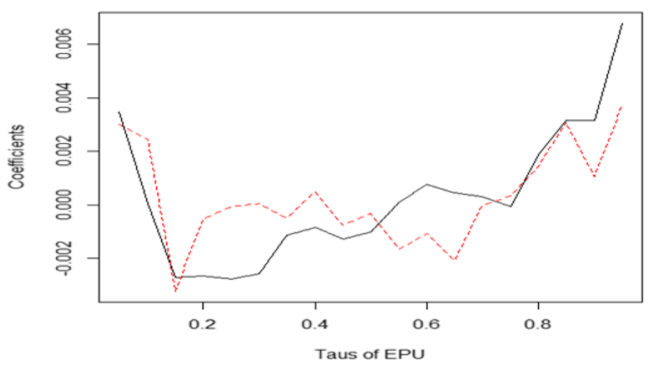

(c4)

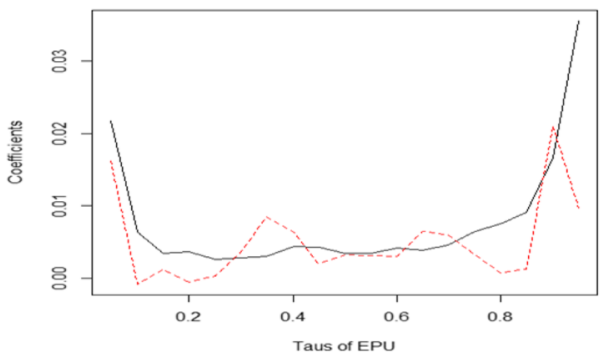

(B4)

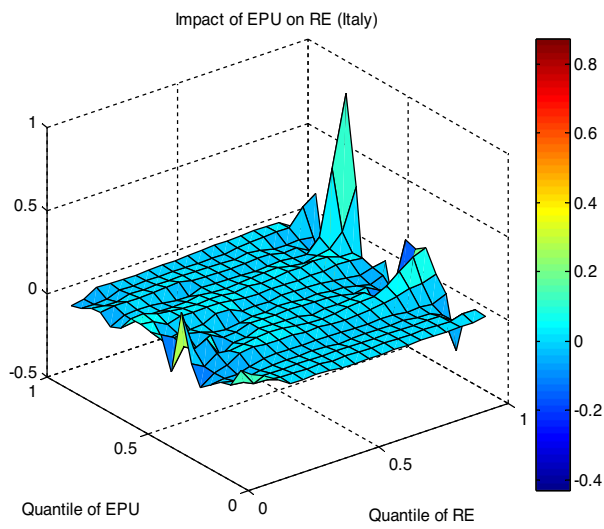

(B4)

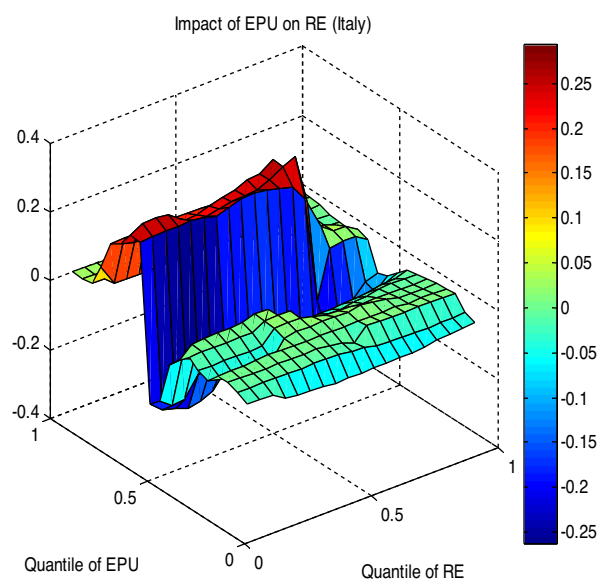

(b4)

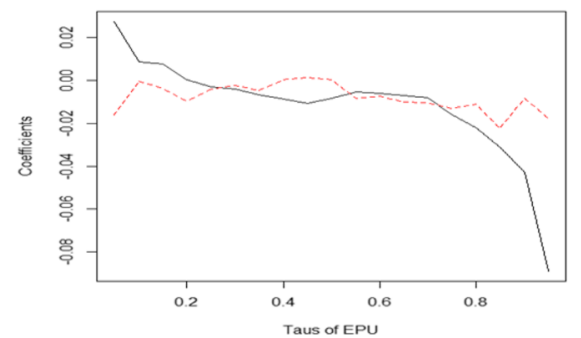

(b4)

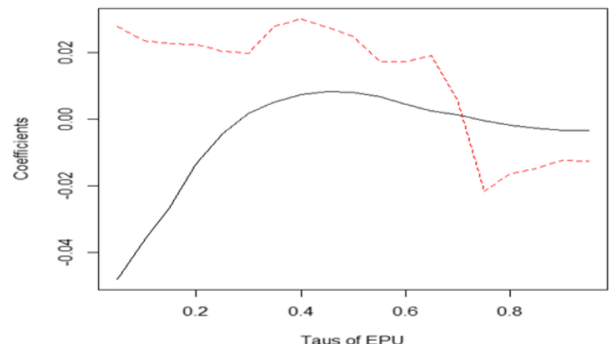

Fig. 4 (A4-D4) QQ estimates of the slope coefficient. (a4-d4) Quantile regression and QQ estimates 
(A5)

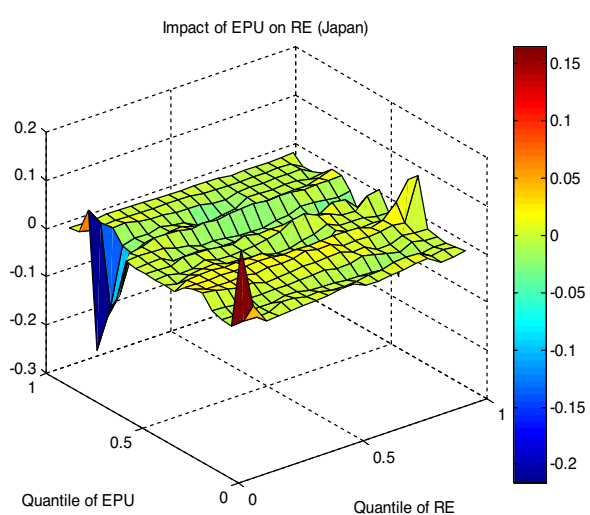

(C5)

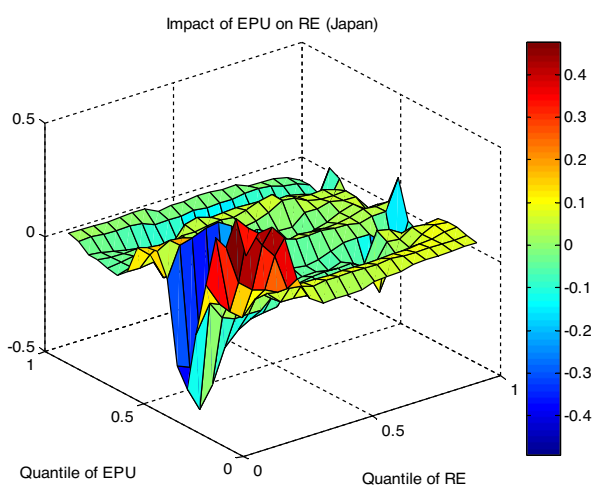

(a5)

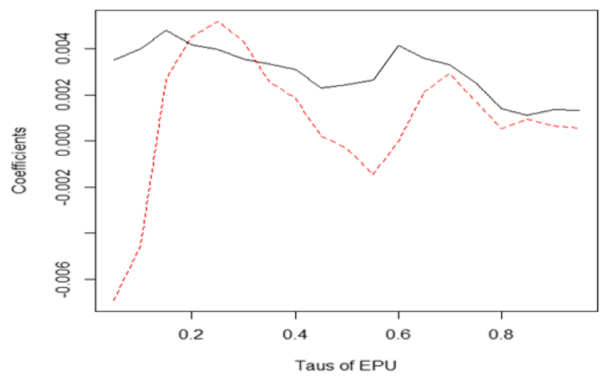

(c5)

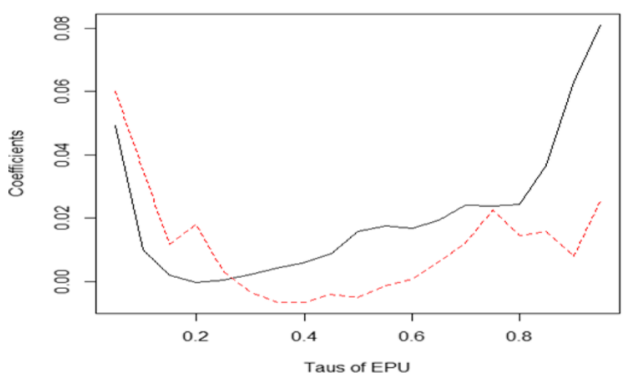

(B5)

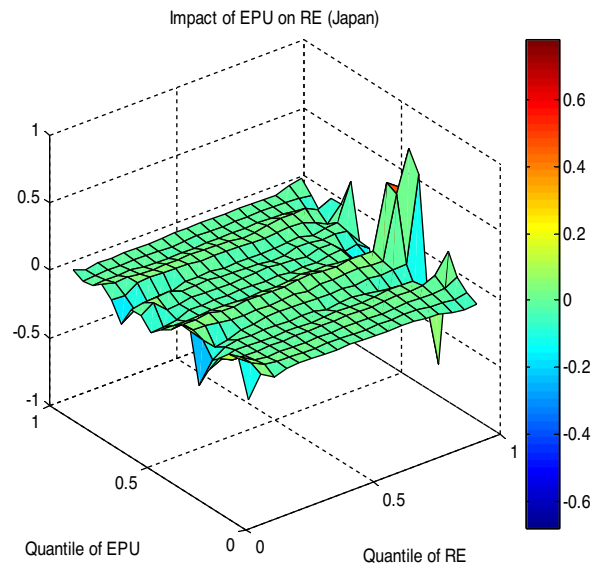

(D5)

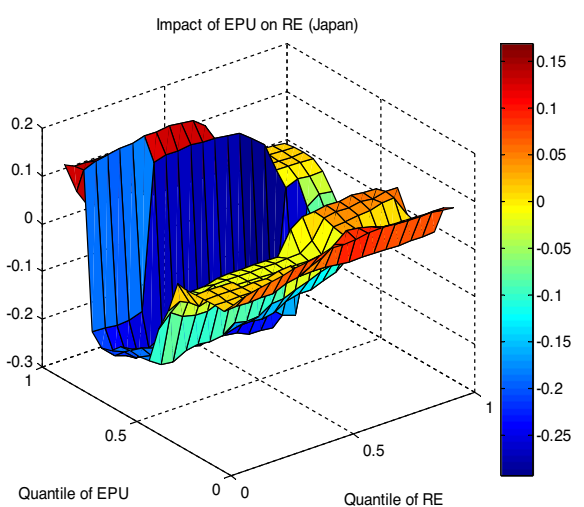

(b5)

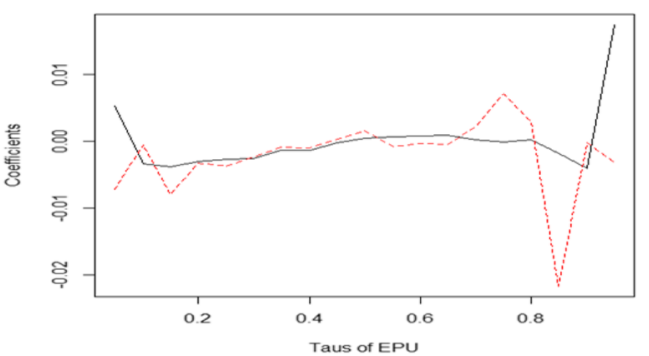

(d5)

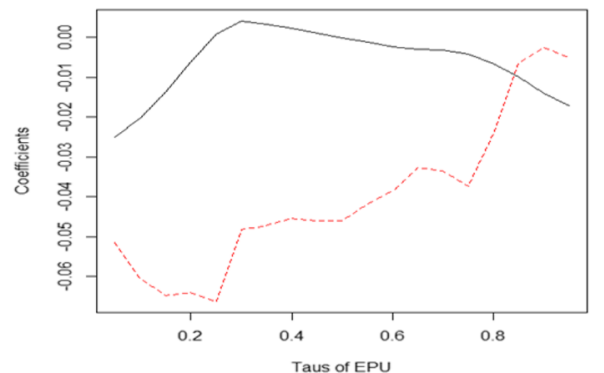

Fig. 5 (A5-D5) QQ estimates of the slope coefficient. (a5-d5) Quantile regression and QQ estimates 
proportional response of RE to EPU. Moreover, EPU has a negative impact on RE in the upper to medium quantiles of $(0.70-0.60)$ in the long run.

Figure 5 (a5-d5) highlights the validity outcomes, which confirm that quantile regression and QQ estimates are the same. It shows that the impact of EPU on RE is consistently negative across all quantiles.

Figure 6(A6) illustrates the result for the UK. It shows that EPU has a negative impact on RE in the medium to upper quantiles $(0.50-0.80)$. The country has witnessed several incidents that have produced higher EPUs, which have a devastating impact on the development of RE (Khan et al. 2020b). Renewable energy is unstable during 2010-2015 because of Brexit and institutional reforms, which can influence the behavior of investment decisions (Adedoyin and Zakari 2020; Sun et al. 2021). Similarly, in 2015, the UK government canceled generous subsidies for RE, which can discourage the development of renewables. Figure 6 (B6-D6) exhibits the wavelet decomposed results. It expresses that EPU has a negative impact on RE in the short run in medium quantiles $(0.60-0.70)$ and the lower to medium quantiles $(0.35-0.50)$ in the mid-run. We find the positive impact of EPU on RE in the lower to upper quantiles $(0.20-0.70)$ in the long run to indicate that uncertainty may be useful for RE development.

Figure 6 (a6-d6) highlights the validity outcomes, which approve that quantile regression and QQ slope coefficients are the same. It shows that the impact of EPU on RE is negative across all the quantiles, except for the long term.

Figure 7(A7) exhibits results for the USA. EPU has a negative effect on RE in medium quantiles $(0.70-0.50)$ in the short run. The USA is the second-largest market for RE in the world driven by supportive government policies at both state and the federal levels (Eryilmaz and Homans 2016). Meanwhile, it is expected that federal tax incentives and state-level policies will support the distribution of solar panels. However, due to the proposed federal tax reform, international trade, and energy policies, EPU may impact RE. From 2009 to 2015 , many policy changes related to RE have occurred in the USA, including suspension of existing or discontinued policies. President Obama's climate action plan aims to reduce carbon emissions, accelerate the energy transition, and promote investment in RE (Burns 2019). During this period, a record number of policy changes have different effects on RE. President Donald Trump's withdrawal from the Paris Agreement has increased EPU, which may have a negative impact on RE. Figure 7 (B7-D7) exhibits the wavelet results. This shows that EPU has a negative impact on RE in the upper quantile $(0.90-0.85)$ in the short term, which shows that higher EPU will cause greater disturbance in RE in the short term. Similarly, in the mid-term, EPU has a negative impact on RE in the upper and lower quantiles $(0.45-0.85)$. However, in the long run, EPU has a negative impact on RE in the low to high quantiles (0.45-0.75). From 2009 to 2015, the policy is stable, and the new climate change and RE policy changes have declined, which is conducive to the development of RE in the USA.

Figure 7 (a7-d7) highlights the validity outcomes, which confirm that quantile regression and QQ estimates are the same. It shows that the effect of EPU on RE is consistently negative across all the quantiles.

We summarize our study as follows. First, the results suggest that EPU has a negative impact on RE in the G7 countries across all the quantiles. The results are in line with the studies of Balcilar et al. (2019), Burns (2019), Sendstad and Chronopoulos (2020), and Appiah-Otoo(2021) that conclude that EPU hurts the macroeconomy that hampers investment and ultimately leads to affect RE. Moreover, the policymakers are hesitant to implement RE policies because of growing uncertainties. The retraction or provision increases or decreases the encouragement for investment. Second, EPU has a significant impact on RE, mostly in the upper quantile, which suggests that high uncertainty in these adversely influences RE rapidly. Wei et al. (2021) support the finding which found that EPU rapidly increases and affects economic activities, and ultimately, this economic shock is reflected in RE. Lastly, the outcome reveals the heterogeneity across these countries and in quantiles. Furthermore, the coefficients indicate the varying impacts of EPU on RE in these countries. The relationship is very weak in some countries such as Canada and Japan. The association coefficient displays that when the relationship changes from short to long term, the influence decreases in Germany. In contrast, the impact increases in Italy, Japan, the UK, and the USA when the relationship changes from short to long run.

\section{Conclusion and policy implications}

This study uses the wavelet QQ method to evaluate EPU impact on RE in G7 countries. The results recommend that EPU has a negative impact on RE across all quantiles, suggesting that EPU adversely affects the macroeconomy that hampers investment and eventually leads to a decline in RE growth. EPU has a significant impact on RE mostly in the upper quantile, which suggests that high uncertainty in these adversely influences RE rapidly. Moreover, the coefficients indicate the varying effects of EPU on RE, and the relationship is very weak in Canada and Japan. Similarly, France and the UK reveal both the negative and positive effects of EPU on RE in the middle-upper quantile. The relationship coefficient shows the impact decreases in Germany when the relationship changes from short to long term. On the contrary, the impact increases in Italy, Japan, the UK, and the USA when the relationship changes from short to long run.

We list the policy implication of this study as follows. First, the result shows that EPU has a negative impact on RE, which 
(A6)

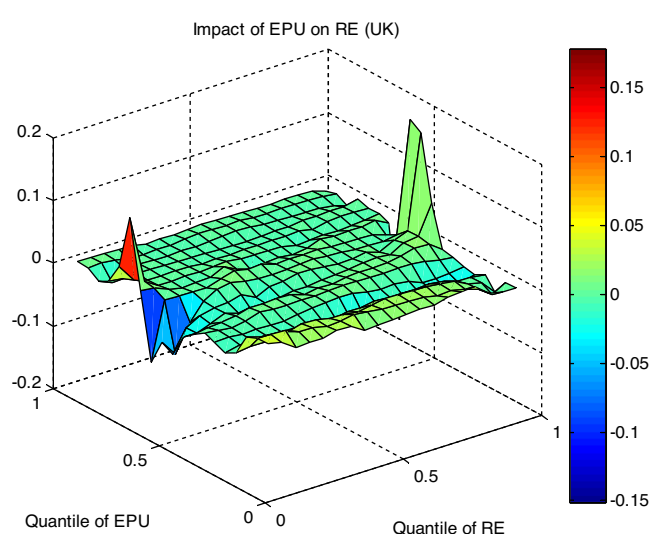

(C6)

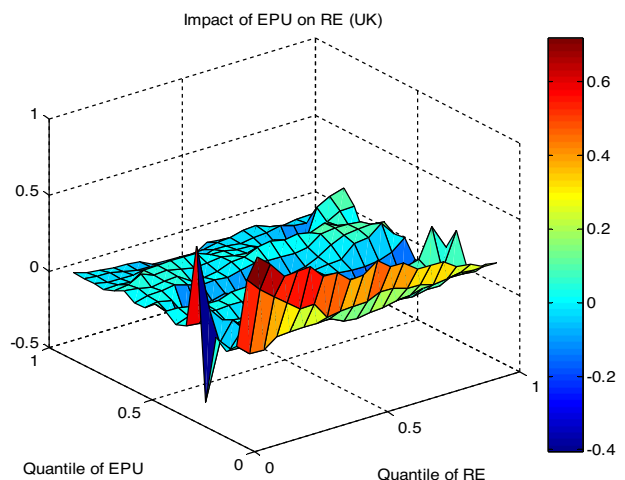

(a6)

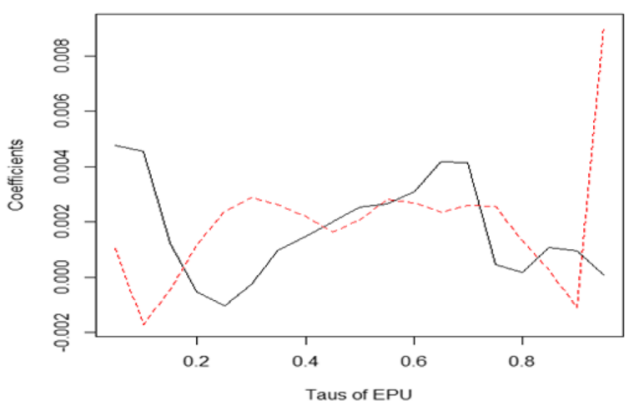

(c6)

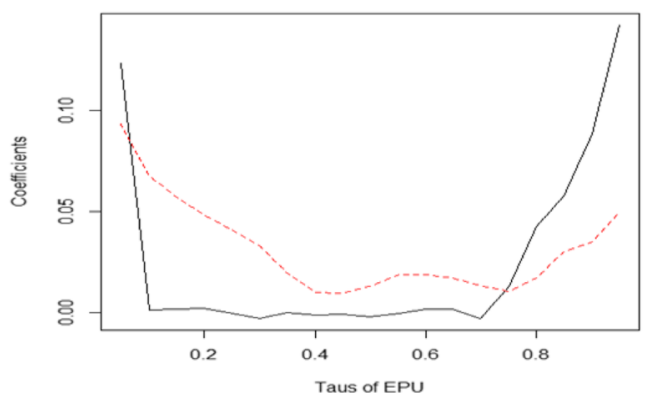

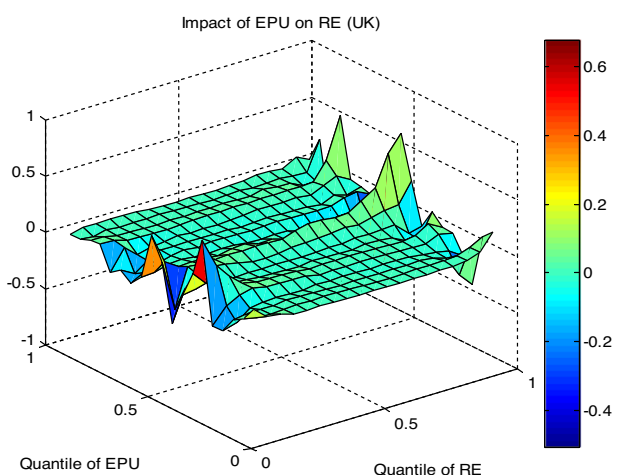

(D6)

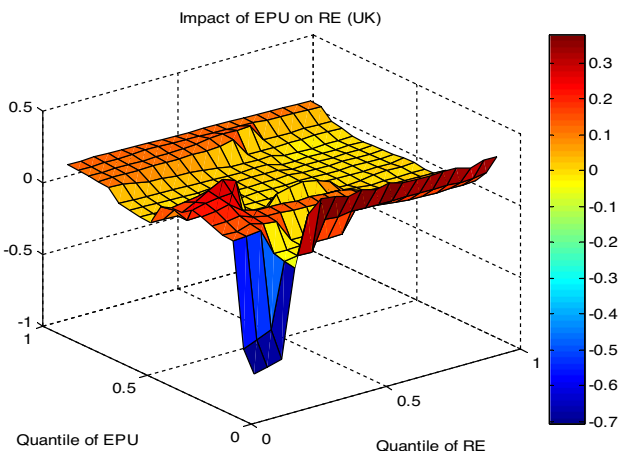

(b6)

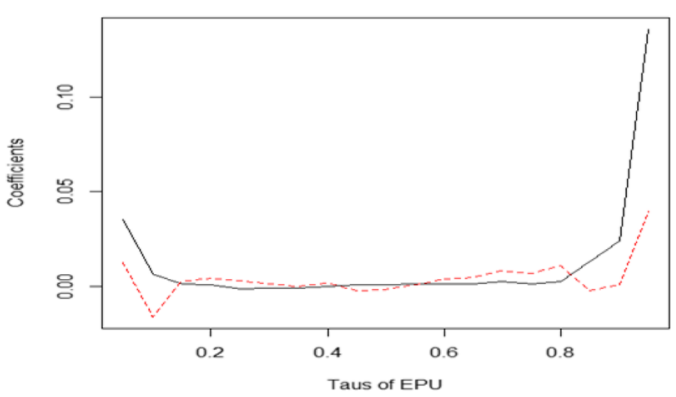

(d4)

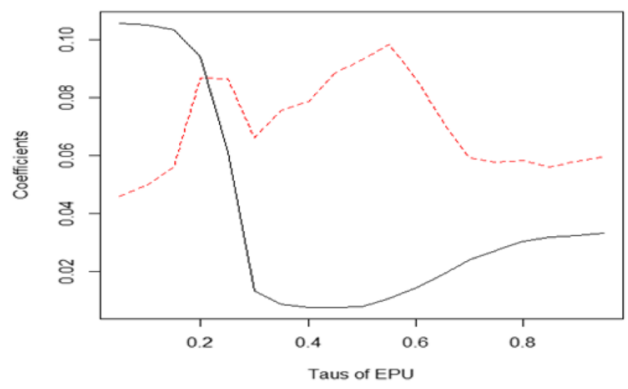

Fig. 6 (A6-D6) QQ estimates of the slope coefficient. (a6-d6) Quantile regression and QQ estimates 
(A7)

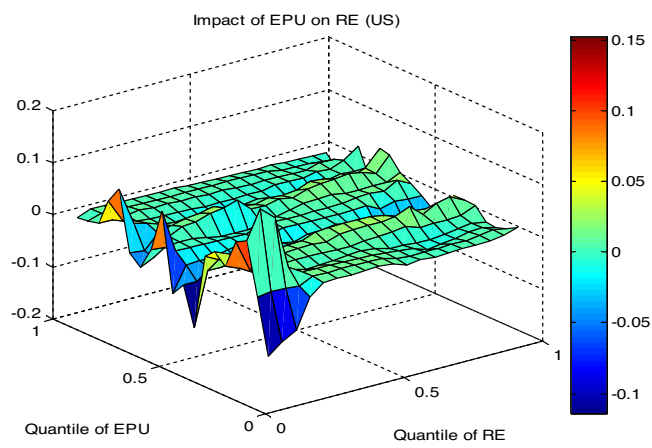

(C7)

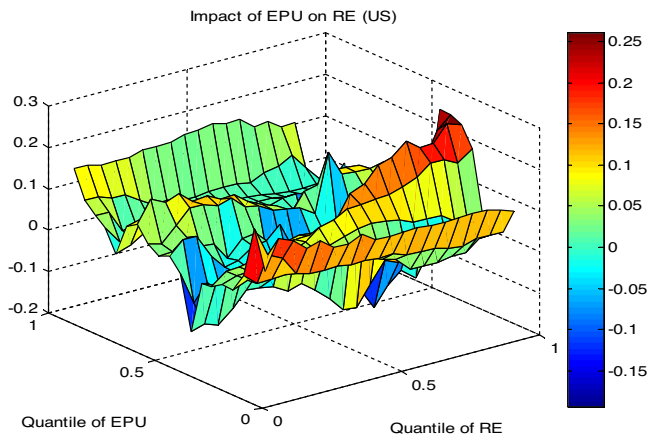

(a7)

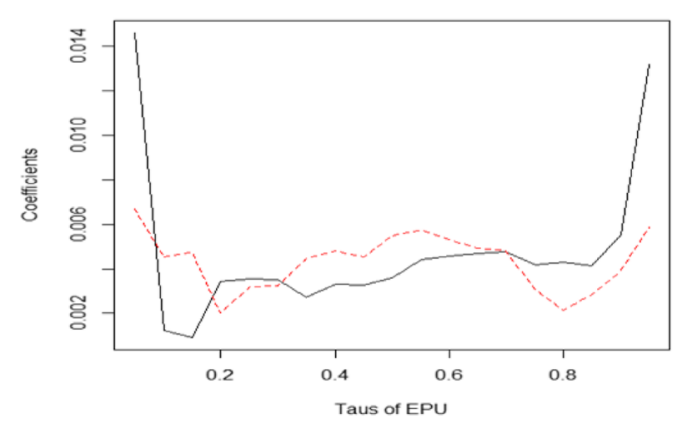

(c7)

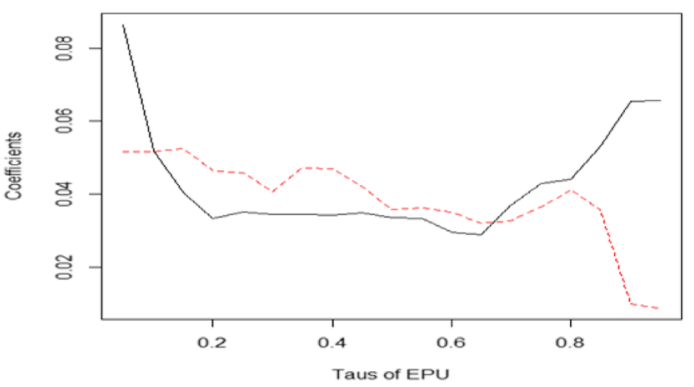

(B7)

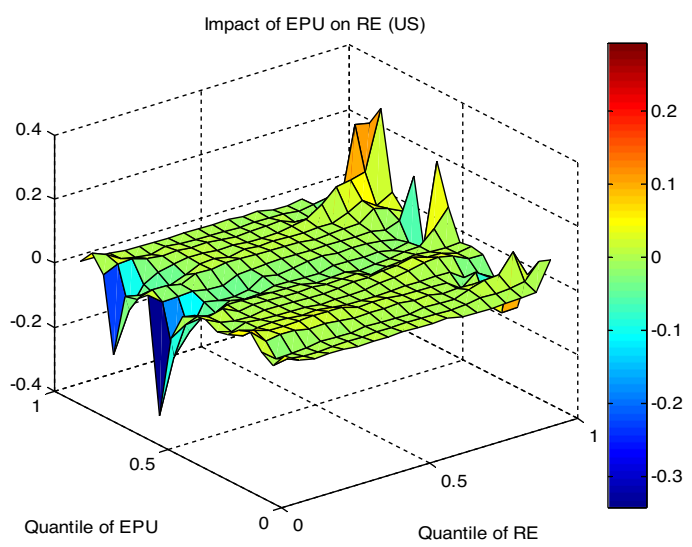

(D7)

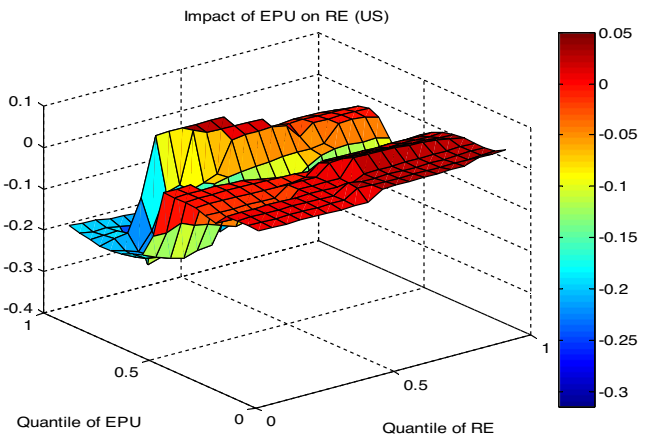

(b7)

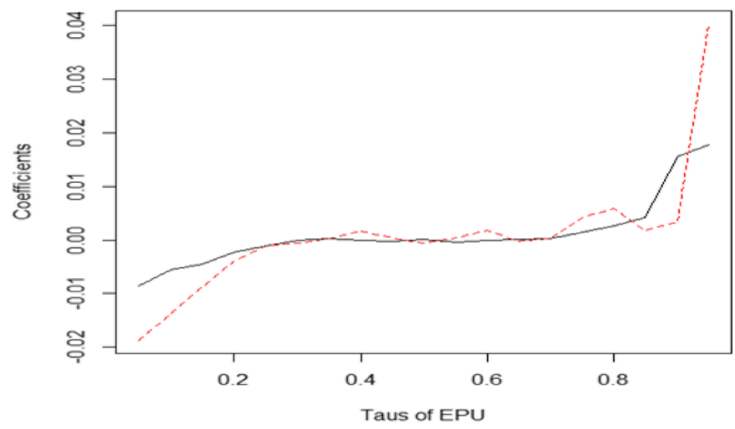

(d7)

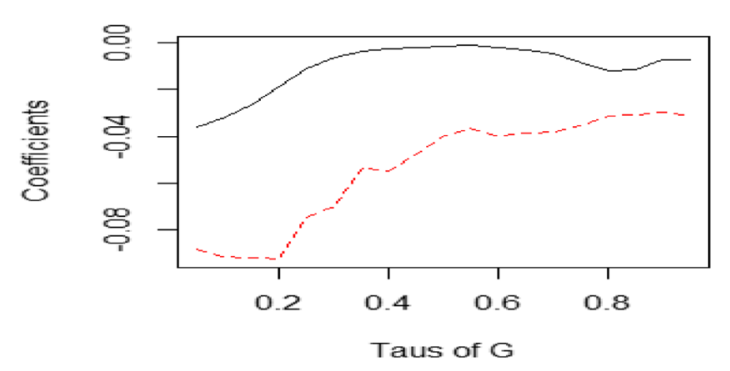

Fig. 7 (A7-D7) QQ estimates of the slope coefficient. (a7-d7) Quantile regression and QQ estimates 
implies that increasing uncertainty leads to threatening RE development in these countries. Therefore, sustainable development of RE requires higher economic stability, which is possible if the government devises the future policy by involving all stakeholders. Likewise, the abrupt policy changes may discourage the investors, which can have a negative impact on RE development. Similarly, the government of these countries has extended subsidies for RE development, and the withdrawal of such facilities adversely influences RE growth. Second, the finding reveals a higher influence of EPU on $\mathrm{RE}$ in the short run, which suggests that uncertainty is more detrimental in the short term. Therefore, the complete information about the planning, execution, and modifications of the economic policies should be readily shared with all the participants. Similarly, the governments should provide a shock absorber facility to investors in case of an unexpected external crisis. Lastly, the country dynamics should be considered while devising renewable energy policy because of heterogeneous results. Thus, the policymakers are cognizant of the policy stability in the process of policy formulation for RE. Similarly, these countries should introduce policies to increase RE shares in the energy mix, which will provide a secure energy supply for sustainable economic development. The regulatory and policy risks will play a considerable role in RE investment and development, which needs to be considered in risk assessment. We can extend this study in the future by considering the impact of the COVID-19 impact on renewable energy in the G7 countries. The crisis has suffered the entire economy, and the energy prices have been hit hard. Similarly, the financial constraints caused by the crisis have threatened or slowed down RE growth around the world. Thus, the investigation of RE in the context of the COVID-19 uncertainty can be a useful contribution in the future.

Author contribution Khalid Khan: idea formulation, data testing analysis, and writing. Chi-Wei Su: methodology, review, and policy implication. Ran Tao: data analysis and editing. Muhammad Umar: data testing, editing, and writing.

Data availability The datasets used and/or analyzed during the current study are available from the corresponding author on reasonable request.

\section{Declarations}

Ethical approval The study has no ethical issue problem related to any person or organization.

\section{Consent to participate Not applicable.}

Consent for publication The author agreed freely to publish the present work in this journal.

Competing interests The authors declare no competing interests.

\section{References}

Abadie LM, Chamorro JM (2014) Valuation of wind energy projects: a real options approach. Energies 7(5):3218-3255

Adedoyin FF, Zakari A (2020) Energy consumption, economic expansion, and $\mathrm{CO}_{2}$ emission in the UK: the role of economic policy uncertainty. Sci Total Environ 738:140014

Ahmed Z, Cary M, Shahbaz M, Vo XV (2021) Asymmetric nexus between economic policy uncertainty, renewable energy technology budgets, and environmental sustainability: evidence from the United States. J Clean Prod 127723.

Appiah-Otoo I (2021) Impact of economic policy uncertainty on renewable energy growth. Energy Research Letters 2(1):19444

Balcilar M, Roubaud D, Shahbaz M (2019) The impact of energy market uncertainty shocks on energy transition in Europe. Energy J 40(01): 55-79

Barradale MJ (2010) Impact of public policy uncertainty on renewable energy investment: wind power and the production tax credit. Energy Policy 38(12):7698-7709

Burns K (2019) Exploring the relationship between energy policy uncertainty and investment in renewable energy. In Local Energy, Global Markets, 42nd IAEE International Conference. International Association for Energy Economics

Caldara D, Iacoviello M (2018) Measuring geopolitical risk. FRB International Finance Discussion Paper. (1222)

Cao H, Guo L, Zhang L (2020) Does oil price uncertainty affect renewable energy firms' investment? Evidence from listed firms in China. Finance Research Letters 33:1-7

Chen C, Xue B, Cai G, Thomas H, Stückrad S (2019) Comparing the energy transitions in Germany and China: synergies and recommendations. Energy Report 5:1249-1260

Chronopoulos M, Hagspiel V, Fleten SE (2016) Stepwise green investment under policy uncertainty. Energy J 37(4):87-108

Crowley PM (2007) A guide to wavelets for economists. J Econ Surv 21(2):207-267

Dalby PA, Gillerhaugen GR, Hagspiel V, Leth-Olsen T, Thijssen JJ (2018) Green investment under policy uncertainty and Bayesian learning. Energy 161:1262-1281

Eryilmaz D, Homans FR (2016) How does uncertainty in renewable energy policy affect decisions to invest in wind energy? Electr $\mathbf{J}$ 29(3):64-71

Fabrizio KR (2013) The effect of regulatory uncertainty on investment: evidence from renewable energy generation. J Law Econ Org 29(4): 765-798

Gatzert N, Vogl N (2016) Evaluating investments in renewable energy under policy risks. Energy Policy 95:238-252

Graps A (1995) An introduction to wavelets. IEEE Comput Sci Eng 2(2): $50-61$

Harrabin R (2016) Investors deterred by energy policy uncertainty. BBC News. 15 June 2016. Available at: https://www.bbc.com/news/ business-36468409

Hagspiel V, Nunes C, Oliveira C, Portela M (2021) Green investment under time-dependent subsidy retraction risk. J Econ Dyn Control 126:103936

Khan K, Su CW, Tao R (2020a) Does oil prices cause financial liquidity crunch? Perspective from geopolitical risk. Defence and Peace Economics 32:1-13

Khan K, Su CW, Xiao YD, Zhu H, Zhang X (2020b) Trends in tourism under economic uncertainty. Tour Econ 27:841-858

Khan K, Sun J, Derindere Koseoglu S, Rehman AU (2021) Revisiting bitcoin price behavior under global economic uncertainty. SAGE Open 11(3):21582440211040411

Liu T, Nakajima T, Hamori S (2021) The impact of economic uncertainty caused by COVID-19 on renewable energy stocks. Empir Econ 121. 
Livingston D (2016) The G7 climate mandate and the tragedy of horizons. Carnegie Endowment for International Peace. Washington, D.C.

Mallat SG (1989) A theory for multiresolution signal decomposition: the wavelet representation. IEEE transactions on pattern analysis and machine intelligence 11(7):674-693

Monfort A, Renne J, Rüffer R, Vitale G (2003) Is economic activity in the G7 synchronized? Common shocks versus spillover effects. CEPR, London

Percival DB, Mofjeld HO (1997) Analysis of subtidal coastal sea level fluctuations using wavelets. J Am Stat Assoc 92(439):868-880

Pirgaip B, Dinçergök B (2020) Economic policy uncertainty, energy consumption and carbon emissions in G7 countries: evidence from a panel Granger causality analysis. Environ Sci Pollut Res 27: 30050-30066

Qin M, Su CW, Tao R (2021) BitCoin: a new basket for eggs? Econ Model 94:896-907

Rechsteiner R (2021) German energy transition (Energiewende) and what politicians can learn for environmental and climate policy. Clean Techn Environ Policy 23(2):305-342

Sendstad LH, Chronopoulos M (2020) Sequential investment in renewable energy technologies under policy uncertainty. Energy Policy 137:111152

Sim N, Zhou H (2015) Oil prices, U.S. stock return, and the dependence between their quantiles. Journal of Banking \& Finance 55:1-8

Sohail MT, Xiuyuan Y, Usman A, Majeed MT, Ullah S (2021) Renewable energy and non-renewable energy consumption: assessing the asymmetric role of monetary policy uncertainty in energy consumption. Environ Sci Pollut Res 1-10

Sun TT, Su CW, Mirza N, Umar M (2021) How does trade policy uncertainty affect agriculture commodity prices? Pac Basin Financ J 66:101514

Su CW, Li X, Tao R (2019a) How does economic policy uncertainty affect prices of housing? Evidence from Germany. Argumenta Oeconomica 42(1):131-153

Su CW, Khan K, Tao R, Nicoleta-Claudia M (2019b) Does geopolitical risk strengthen or depress oil prices and financial liquidity? Evidence from Saudi Arabia. Energy 187:116003

Su CW, Khan K, Tao R, Umar M (2020) A review of resource curse burden on inflation in Venezuela. Energy 204:117925
Su CW, Yuan X, Tao R, Umar M (2021a) Can new energy vehicles help to achieve carbon neutrality targets? J Environ Manag 297:113348

Su CW, Song Y, Umar M (2021b) Financial aspects of marine economic growth: from the perspective of coastal provinces and regions in China. Ocean \& Coastal Management 204:105550

Su CW, Cai XY, Qin M, Tao R, Umar M (2021c) Can bank credit withstand falling house price in China? International Review of Economics \& Finance 71:257-267

Su CW, Qin M, Zhang XL, Tao R, Umar M (2021d) Should bitcoin be held under the U.S. partisan conflict? Technol Econ Dev Econ 27: 511-529

Tao R, Umar M, Naseer A, Razi U (2021a) The dynamic effect of ecoinnovation and environmental taxes on carbon neutrality target in emerging seven (E7) economies. J Environ Manag 299:113525

Tao R, Su CW, Xiao Y, Dai K, Khalid F (2021b) Robo advisors, algorithmic trading and investment management: wonders of fourth industrial revolution in financial markets. Technol Forecast Soc Chang 163:120421

Tao R, Su CW, Yaqoob T, Hammal M (2021c) Do financial and nonfinancial stocks hedge against lockdown in COVID-19? An event study analysis. Economic Research-Ekonomska Istraživanja 1-22.

Torrence C, Webster PJ (1999) Interdecadal changes in the ENSOmonsoon system. Journal of Climate 12(8):2679-2690

Vakulchuk R, Overland I, Scholten D (2020) Renewable energy and geopolitics: a review. Renew Sust Energ Rev 122:109547

Wang KH, Su CW, Lobonț OR, Umar M (2021) Whether crude oil dependence and $\mathrm{CO}_{2}$ emissions influence military expenditure in net oil-importing countries? Energy Policy 153:112281

Wei W, Hu H, Chang CP (2021) Economic policy uncertainty and energy production in China. Environ Sci Pollut Res 1-24

Yahya M, Oglend A, Dahl RE (2019) Temporal and spectral dependence between crude oil and agricultural commodities: a wavelet-based copula approach. Energy Economics 80:277-296

Zhu D, Mortazavi SM, Maleki A, Aslani A, Yousefi H (2020) Analysis of the robustness of energy supply in Japan: role of renewable energy. Energy Reports 6:378-391

Publisher's note Springer Nature remains neutral with regard to jurisdictional claims in published maps and institutional affiliations. 